\title{
Masers as evolutionary tracers of high-mass star formation
}

\author{
Shari L. Breen ${ }^{1}$ and Simon P. Ellingsen ${ }^{2}$ \\ ${ }^{1}$ CSIRO Astronomy and Space Science, Australia Telescope National Facility, PO Box 76, \\ Epping NSW 1710, Australia \\ email: Shari.Breen@csiro.au \\ ${ }^{2}$ School of Mathematics and Physics, University of Tasmania, GPO Box 37, Hobart, Tasmania \\ 7000, Australia
}

\begin{abstract}
Determining an evolutionary clock for high-mass star formation is an important step towards realising a unified theory of star formation, as it will enable qualitative studies of the associated high-mass stars to be executed. We have carried out detailed studies of a large number of sources suspected of undergoing high-mass star formation and have found that common maser transitions offer the best opportunity to determine an evolutionary scheme for these objects. We have investigated the relative evolutionary phases of massive star formation associated with the presence or absence of combinations of water, methanol and main-line hydroxyl masers. The locations of the different maser species have been compared with the positions of $1.2 \mathrm{~mm}$ dust clumps, radio continuum, GLIMPSE point sources and Extended Green Objects. Comparison between the characteristics of coincident sources has revealed strong evidence for an evolutionary sequence for the different maser species in high-mass star formation regions. We present our proposed sequence for the presence of the common maser species associated with young highmass stars and highlight recent advances. We discuss future investigations that will be made in this area by comparing data from the Methanol Multibeam (MMB) Survey with chemical clocks from the Millimetre Astronomy Legacy Team $90 \mathrm{GHz}$ (MALT90) Survey.
\end{abstract}

Keywords. masers - ISM: molecules - stars: formation

\section{Introduction}

The process through which high-mass stars form is one of the hottest topics in modern astrophysics, with implications for fields as diverse as galactic evolution and the epoch of reionization. Masers are one of the best, if not the best signpost of young high-mass star formation regions. They are relatively common, intense and because they arise at centimetre wavelengths, are not affected by the high extinction that plagues observations in other wavelength ranges. To date progress has been slow towards the overall goal of utilising masers as tools to study star formation, however, the pace of advancement has recently accelerated. The proliferation of complementary high-resolution observations of star formation regions at millimetre through mid-infrared wavelengths means that this trend is likely to continue.

One of the difficulties in understanding the process through which high mass stars form is the lack of good sequential signposts in identifying different evolutionary stages of star formation, especially during the early stages while the young stellar objects are still embedded in their natal molecular clouds. Some types of masers are very common in high-mass star formation regions, for example, the $22 \mathrm{GHz}$ water, 6.7 and $12.2 \mathrm{GHz}$ methanol and $1.6 \mathrm{GHz}$ hydroxyl transitions, while others are much more rare, such as the $37.7 \mathrm{GHz}$ and $107 \mathrm{GHz}$ methanol masers. Masers are created under a very specific set of physical conditions (e.g. Cragg et al. 2005) so those transitions which are common 
and strong, likely trace conditions that arise often and persist, while those that are rare likely trace rare or short-lived phases in the evolution of these regions.

Historically, attempts to construct a sequential timeline of the different common maser species in high-mass star formation regions have uncovered mixed results, primarily due to being based on heavily biased samples or small numbers of special sources. Furthermore, the observations often had poor spatial resolution and/or poor sensitivity, inducing confusion amongst sources with small angular separations, or failing to detect weak maser emission. There are some exceptions such as the early work by Forster \& Caswell (1989) who showed that water masers appear prior to hydroxyl masers. Other works such as those by Walsh et al. (1998), Garay \& Lizano (1999), Ellingsen (2006) and Fontani et al. (2010) have provided further strong evidence that masers make excellent evolutionary probes, but their samples are plagued to varying degrees by strong selection biases which limits the meaningfulness of their results.

At the last IAU maser conference in Alice Springs, Australia, Ellingsen et al. (2007) presented a 'straw man' evolutionary sequence for masers in high-mass star formation regions using a combination of new results and previously established facts from the literature. Here we outline advances towards establishing a robust evolutionary timeline for high-mass star formation regions that have been made since then.

\section{Advances towards an accurate maser evolutionary timeline}

Recently a great deal of progress has been made toward a robust maser evolutionary timeline, owing to the abundance of new high-sensitivity, high-resolution, large maser datasets together with complementary data. Chief amongst these important advances is the Methanol Multibeam survey (described in Section 2.1 and in more detail in Green et al. (2009)). Other contributors include $12.2 \mathrm{GHz}$ methanol maser observations towards the MMB sources (Breen et al. 2010a, 2011, 2012) and new water maser catalogues with accurately determined positions (Breen et al. 2010b; Breen \& Ellingsen 2011).

\subsection{The Methanol Multibeam Survey (MMB)}

The MMB is a complete survey for $6.7 \mathrm{GHz}$ methanol masers within the Galactic plane (Green et al. 2009). These masers are especially useful as they exclusively trace sites of high-mass star formation (e.g. Minier et al. 2003) and trace the systemic velocities (e.g. Szymczak et al. 2007) of the regions that they are associated with, making them excellent tools for investigating not only the kinematics and the physical conditions of the regions that they are tracing, but also aspects like Galactic structure (Green et al. 2011).

The southern hemisphere component of the survey has been completed using the Parkes radio telescope. The location of Parkes allowed about $60 \%$ of the Galactic plane to be surveyed, from a longitude of $186^{\circ}$, through the Galactic centre, to a longitude of $60^{\circ}$, with a latitude extent of $\pm 2^{\circ}$. The survey was completed in a scanning mode with a purpose built seven beam receiver which dramatically reduced the time required to complete the

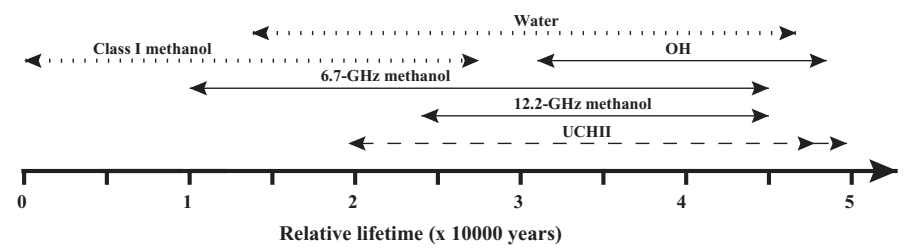

Figure 1. Proposed evolutionary timeline for the common maser species found towards high-mass star formation regions (Breen et al. 2010). 
observations. During the course of the survey $\sim 1000$ methanol masers $(3 \sigma$ sensitivity of $0.7 \mathrm{Jy}$ ) were detected, of which $\sim 40 \%$ are new detections, pinpointing sites of highmass star formation. All detected sources without previously delivered accurate positions were followed up with an interferometer, chiefly the Australia Telescope Compact Array, resulting in positional accuracies of at least 0.4 arcsec for all detected masers.

The MMB maser catalogues in the longitude range: $186^{\circ}$ (through the Galactic centre) to $20^{\circ}$ have now been published and include the accurate source positions (Green et al. 2012; Caswell et al. 2011; Caswell et al. 2010; Green et al. 2010).

\subsection{Constructing an improved maser timeline}

The first quantitative timeline (see Fig. 1) for the common maser species in high-mass star formation was created by Breen et al. (2010). Using new, large samples of methanol masers at 6.7 (from the MMB survey) and $12.2 \mathrm{GHz}$, water masers and $\mathrm{OH}$ masers (Caswell 1998), together with mid-infrared (GLIMPSE), $1.2 \mathrm{~mm}$ dust continuum (Hill et al. 2005) and cm radio continuum data (Walsh et al. 1998), Breen et al. (2010, 2011, 2012) showed, through statistical analysis of their data, strong evidence that it is not only the presence or absence of the different maser species that can indicate the evolutionary stage of the high-mass star formation region that they are associated with, but that the properties of those masers can give even finer evolutionary details. Most notably, they show that both the luminosity and velocity range of detected $6.7 \mathrm{GHz}$ and $12.2 \mathrm{GHz}$ methanol and water maser emission increases as the star forming region evolves. The left panel of Fig. 2 shows that the 6.7 and $12.2 \mathrm{GHz}$ methanol masers associated with $\mathrm{OH}$ masers (known to be associated with a later phase of evolution) tend to have higher luminosities. This is a notion supported by comparisons made by Wu et al. (2010) who showed that the most luminous methanol masers were associated with ammonia profiles indicative of more evolved objects than the methanol masers with lower luminosities.

Subsequent work by Ellingsen et al. (2011) used the results of Breen et al. $(2010,2011)$ to show that the presence of rare $37.7 \mathrm{GHz}$ methanol masers may signal the end of the methanol maser phase. The right panel of Fig. 2 shows these $37.7 \mathrm{GHz}$ methanol masers are associated with only the most luminous 6.7 and $12.2 \mathrm{GHz}$ methanol masers, which combined with the rarity of these objects is consistent with them being a short lived phase towards the end of the 6.7 and $12.2 \mathrm{GHz}$ methanol maser lifetime.
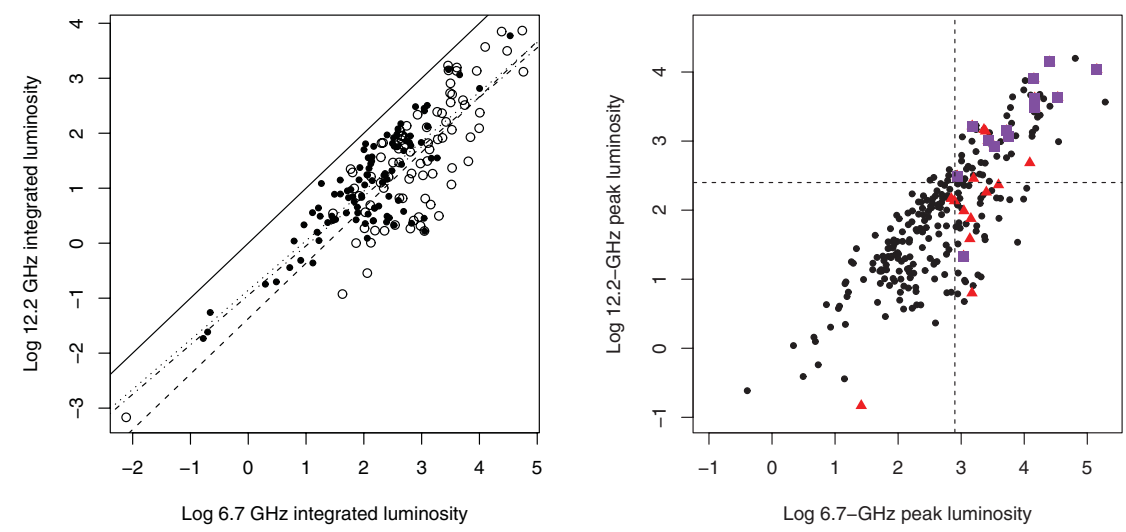

Figure 2. Left: Log $12.2 \mathrm{GHz}$ versus $\log 6.7 \mathrm{GHz}$ integrated luminosities (Breen et al. 2012). Methanol masers associated with $\mathrm{OH}$ masers are shown by unfilled circles and those without are shown by dots. Right: Luminosity of $12.2 \mathrm{GHz}$ versus luminosity of the $6.7 \mathrm{GHz}$ methanol masers (black dots). Sources that also exhibit emission from $37.7 \mathrm{GHz}$ methanol masers are shown by purple squares (Ellingsen et al. 2011). 


\subsubsection{The evolutionary stage traced by water and class I methanol masers}

As noted in Breen et al. (2010), the relative lifetime of water and class I methanol masers could only be estimated from the maser samples at the time. While some progress has been made towards understanding the evolutionary stage (or stages) that these transitions are tracing, such as the tendency of some class I methanol masers to be associated with a later evolution phase (e.g. Voronkov et al. 2010), much larger samples of sources need to be investigated before we can refine the timeline shown in Fig. 1.

\section{Comparisons with Chemical Clocks}

Non-masing molecular transitions have also proved to be useful as signposts of the evolutionary stage of high-mass stars (e.g. Longmore et al. 2007; Lee et al. 2004). Therefore an independent test of our proposed maser evolutionary scenario can be achieved by comparing evolutionary stage estimates derived from maser observations with those deduced from observations of certain molecules (or chemical clocks).

\subsection{The MALT90 Survey}

The Millimetre Astronomy Legacy Team 90 GHz (MALT90) Survey is a large project designed to characterise the physical and chemical evolution of dense cores (see Foster et al. (2011) for a description of the pilot survey). The survey will map more than 2000 cores with the Mopra radio telescope in 16 molecular lines near $90 \mathrm{GHz}$. At this frequency the spectrum is rich in diagnostic lines, spanning a range of excitation energies and densities, revealing distinct physical conditions and different stages in the chemical evolution of each core. The targets were selected from the $870 \mu \mathrm{m}$ ATLASGAL survey (Schuller et al. 2009) of dust continuum emission and cover a wide range of evolutionary states, from pre-stellar cores, to protostellar cores, and finally, cores with HII regions. MALT90 data from the first two observing seasons (1135 cores observed in 2010 and 2011) have been publicly released and can be downloaded from the Australia Telescope Online Archive.

\subsection{Comparing the MMB to MALT90}

MALT90 provides the perfect dataset to test the maser evolutionary timeline. About $25 \%$ of the MALT90 cores observed are coincident with MMB sources, providing a large enough sample for meaningful analysis of the evolutionary stage associated with the masers, and also how this stage fits into the broader picture of star formation. Preliminary comparisons between these data shows that the methanol masers are overwhelmingly associated with MALT90 sources classified as 'protostellar'. Fig. 3 shows a typical example of MALT90 data towards a region associated with a methanol maser, exhibiting strong $\mathrm{N}_{2} \mathrm{H}^{+}$, but no $\mathrm{HCO}^{+}$or $\mathrm{HCN}$. It is such obvious chemical signatures as these that can be used as a 'chemical clock' and test the current maser evolutionary timeline.
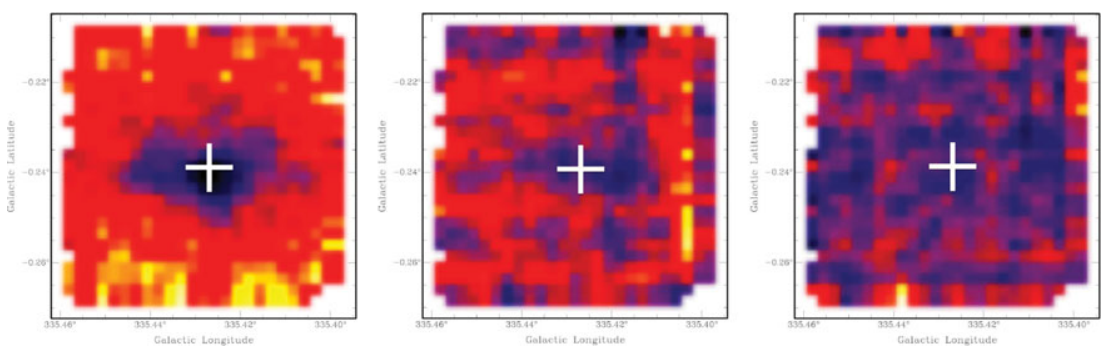

Figure 3. MALT90 maps of $\mathrm{N}_{2} \mathrm{H}^{+}, \mathrm{HCO}^{+}$and $\mathrm{HCN}$ for one source associated with a MMB methanol maser (white cross). 


\section{Conclusions}

Masers provide the best opportunity for a robust and detailed timeline for the evolution of high-mass star formation. We have shown that not only the presence or absence of different maser species can give an indication of the evolutionary stage of the associated object, but that maser properties such as luminosity and velocity range can offer finer evolutionary detail. Investigations of large samples of water and class I methanol masers will allow their position on the current maser evolutionary timeline to be more accurately estimated. Comparisons with molecular data from the MALT90 survey will allow the maser evolutionary timeline to be independently confirmed. Preliminary results show that the $6.7 \mathrm{GHz}$ methanol masers are associated with MALT90 sources with similar evolutionary stage classifications and chemical properties - showing great promise for future detailed investigations.

\section{Acknowledgements}

We acknowledge the contributions made to this work by the MMB and MALT90 survey teams, and in particular: James Caswell, James Green, Gary Fuller, Maxim Voronkov, Jill Rathborne, James Jackson and Jonathan Foster. The Australia Telescope Compact Array, Parkes and Mopra telescopes are part of the Australia Telescope National Facility which is funded by the Commonwealth of Australia for operation as a National Facility managed by CSIRO.

\section{References}

Breen, S. L., Ellingsen, S. P., Caswell, J. L., \& Lewis, B. E., 2010a, MNRAS, 401, 2219

Breen, S. L., Ellingsen, S. P., Caswell, J. L., \& Phillips, C. J., 2010b, MNRAS, 733, 406, 1487

Breen, S. L., Ellingsen, S. P., Caswell, J. L., Green, J. A., et al., 2011, ApJ, 733, 80

Breen, S. L. \& Ellingsen, S. P., 2011, MNRAS, 416, 178

Breen, S. L., Ellingsen, S. P., Caswell, J. L., Green, J. A., et al., 2012, MNRAS, 421, 2511

Caswell, J. L., Fuller, G. A., Green, J. A., Avison, A., et al., 2010, MNRAS, 404, 1029

Caswell, J. L., Fuller, G. A., Green, J. A., Avison, A., et al., 2011, MNRAS, 417, 1964

Cragg, D. M., Sobolev, A. M., \& Godfrey, P. D., 2005, MNRAS, 360, 533

Ellingsen, S. P., 2006, ApJ, 638, 241

Ellingsen S. P., et al., 2007, in Chapman J. M., Baan W. A., eds., Proc. IAU Symp., 242, Astrophysical Masers and their Environments. Cambridge Univ. Press, Cambridge, p. 213

Ellingsen, S. P., Breen, S. L., Sobolev, A. M., Voronkov, M. A., et al., 2011, ApJ, 742, 109

Fontani, F., Cesaroni, R., \& Furuya, R. S., 2010, A\&A, 517, 56

Forster, J. R. \& Caswell, J. L., 1989, A\&A, 213, 339

Foster, J. B., Jackson, J. M., Barnes, P. J., \& Barris, E., 2011, ApJS, 197, 25

Garay, G. \& Lizano, S., 1999, PASP, 111, 1049

Green, J. A., Caswell, J. L., Fuller, G. A., Avison, A. et al., 2009, MNRAS, 392, 783

Green, J. A., Caswell, J. L., Fuller, G. A., Avison, A. et al., 2010, MNRAS, 409, 913

Green, J. A., Caswell, J. L., McClure-Griffiths, N., Avison, A. et al., 2011, ApJ, 733, 27

Green, J. A., Caswell, J. L., Fuller, G. A., Avison, A. et al., 2012, MNRAS, 420, 3108

Lee, J.-E., Bergin, E. A., \& Evanse, N. J., 2004, ApJ, 617, 360

Longmore S. N., et al., 2007, MNRAS, 379, 535

Minier, V., Ellingsen, S. P., Norris, R. P., \& Booth, R. S., 2003, A\&GA, 403, 1095

Schuller, F., Menten, K., Contreras, K. M., \& Wyrowski, F., 2009, A\&A, 504, 415

Szymczak, M., Bartkiewicz, A., \& Richards, A. M. S., 2007, ApJ, 706, 1609

Voronkov, M. A., Caswell, J. L., Ellingsen, S. P., \& Sobolev, A. M., 2010, MNRAS, 405, 2471

Walsh, A. J., Burton, M. G., Hyland, A. R., \& Robinson, G., 1998, MNRAS, 301, 640

Wu, Y. W., Xu, Y., Pandian, J. D., Yang, J., et al., 2010, ApJ, 720, 392 